\title{
Kaon photoproduction off proton
}

\author{
Dalibor Skoupil ${ }^{1,2, \star}$ and Petr Bydžovský ${ }^{1}$ \\ ${ }^{1}$ Nuclear Physics Institute, Czech Academy of Sciencies, Řež near Prague, Czech Republic \\ ${ }^{2}$ Czech Technical University in Prague, Czech Republic
}

\begin{abstract}
We have recently constructed our version of the Regge-plus-resonance (RPR) model and two variants of an isobar model for photoproduction of kaons on the proton, utilizing new experimental data from CLAS, LEPS, and GRAAL collaborations for adjusting free parameters of the models. Higher-spin nucleon (3/2 and 5/2) and hyperon (3/2) resonances were included using the consistent formalism by Pascalutsa and found to play an important role in data description. The set of chosen nucleon resonances in our new isobar models agrees well with the set of the most probable contributing states determined in the Bayesian analysis with the RPR model whilst only 6 out of $10 \mathrm{~N}$ 's selected in the RPR fit of ours overlap with the nucleon resonant states in the Bayesian analysis. Results of two versions of the isobar model are compared to the new version of the RPR model and experimental data in the third-resonance region and their properties are discussed. We place an emphasis on the choice of resonances, the predictions in the forward- and backward-angle region as well as the choice of the hadron form factor.
\end{abstract}

\section{Introduction and methodology}

The study of kaon-hyperon electro- and photoproduction from nucleons in the nucleon resonance region supplies important information about the spectrum of baryon resonances and interactions in hyperon-nucleon systems which arise from Quantum Chromodynamics. A right description of the elementary production process of a $\Lambda$ hyperon is crucial for obtaining dependable predictions of the excitation spectra for production of $\Lambda$ hypernuclei. What is more, the "missing" resonances, which were predicted by the quark models [5] but are still elusive in the pion production or $\pi N$ scattering processes, can be studied by investigating the kaon-hyperon production. In the last decade, experimental data of good quality from the CLAS, LEPS, GRAAL, and MAMI collaborations were produced, which has attracted the attention of many groups of theoretical physicists to this subject.

There are many models of $p\left(\gamma, K^{+}\right) \Lambda$ process at hand, which provide acceptable description of the current experimental data. In our work, we focus on single-channel isobar models and neglect all higher-order effects, e.g. rescattering or an interaction in the final state, assuming that their influence on results is included, to some extent, by means of effective values of the coupling constants fitted to experimental data. This simplifying assumption was adopted in numerous single-channel isobar models, e.g. Saclay-Lyon (SL) [7], Kaon-MAID (KM) [11] and Ghent Isobar model. The short-range physics, manifesting itself via a spatial structure of hadrons, can be simulated by a hadron form factor

^e-mail: skoupil@ujf.cas.cz 
(hff) introduced in the strong interaction vertex. The role of the hff consists also in suppressing the overly strong contributions of Born terms and reducing the high-momentum dependence in exchanges of high-spin resonances [14].

In the isobar model, the amplitude is constructed from effective Lagrangians as a sum of tree-level Feynman diagrams representing the $s-, t$, and $u$-channel exchanges of hadrons in their ground state (Born terms) and various resonances (non Born terms). It is only the exchanges of nucleon resonances in the $s$ channel that make a resonant structure in the observables; the other diagrams constitute the background part of the amplitude as the corresponding poles of theirs are far from the physical region.

The hybrid Regge-plus-resonance (RPR) model, constructed by the Ghent group [6, 8], allows to describe the kaon-hyperon photo- and electroproduction from the threshold up to energies well above the resonance region $\left(E_{\gamma} \approx 16 \mathrm{GeV}\right)$. This model combines merits of the Regge model [9], which is suitable for description above the resonance region, with the isobar model suited for description of the resonance region. The non resonant part of the amplitude, which is a smooth function of energy, is constructed by exchanges of two degenerate kaon trajectories with only three free parameters. The resonant part of the amplitude is then modelled by means of the $s$-channel exchanges of nucleon resonances whose contributions are made to fade away beyond the resonance region by a strong hff. Thanks to the economical description of the non resonant part of the amplitude, the number of free parameters is significantly reduced in comparison with the isobar model.

Since the kaon production takes place in the third-resonance region, where many nucleon and hyperon higher-spin states might couple to kaon-hyperon channels, the contributions of higher-spin baryon resonances are particularly important in the isobar models. The theoretical description of the interacting baryon fields with spin higher than $1 / 2$ causes problems because of the presence of non physical lower-spin components in the Rarita-Schwinger (RS) field [2]. Provided the RS field is off its mass shell, the non physical lower-spin components of the RS field may participate in the interaction which is then entitled "inconsistent". These problems were eliminated by Pascalutsa who formulated a "consistent" interaction theory for massive spin-3/2 fields demanding invariance of the spin-3/2 interaction vertices under the local U(1) gauge transformation of the RS field [13]. In the consistent theory, the interaction vertices are transverse, which leads to removal of all non physical contributions. This formalism was generalized to arbitrary high spin by the Ghent group [15] and is exploited in this work. The gauge invariance of the interaction leads to a relatively strong momentum dependence of the amplitude, which increases with rising spin of the RS field and regularizes the amplitude. However, the strong dependence on the momentum creates some non physical structures in the energy dependence of the cross section, which then need to be cut off with help of strong hff particularly above the resonance region.

\section{Results}

The free parameters of the isobar and RPR models, namely the coupling constants and cutoff parameters of the hff, have been fitted to differential-cross-section and hyperon-polarization data. In the fitting procedure of isobar-model parameters, the CLAS data sets were limited in energy to $W<2.4 \mathrm{GeV}$ and $W<2.2 \mathrm{GeV}$ for differential cross section and hyperon polarization, respectively, whereas for the adjustment of RPR free parameters these data sets were used in their entirety. In comparison to the isobar model, we therefore had less free parameters to optimize and more data available when we went through the fitting procedure of the RPR model.

For the isobar model, many combinations of nucleon and hyperon resonances have been considered and the best solutions have been found: these are the BS1 and BS2 models both with $\chi^{2} /$ n.d.f. $=1.64$. In these models, the set of chosen nucleon resonances largely overlaps with the 
set found in the Bayesian analysis with the RPR model [8], which suggests that the present data can already fix the resonant part of the amplitude well. Besides others, we confirm the importance of the missing resonances $P_{13}(1900)$ and $D_{13}(1875)$ for the data description in the $K^{+} \Lambda$ channel but we have replaced the $P_{11}(1880)$ resonance with a $F_{15}(1860)$ resonance which was recently included in the Particle Data Group tables [10]. The BS models differ from one another considerably in the choice of hyperon resonances, which contribute to the non resonant part, and in a form of the hff. While the BS1 model was gained using a multidipole hff, the BS2 model was obtained exploiting a dipole shape of the form factor. The main benefit of the presence of hyperon resonances with spin $3 / 2$ lies in the reduction of the coupling parameters of spin-1/2 hyperon resonances which generally tend to be large. The contributions of spin-3/2 hyperon resonances is perceptible particularly at backward kaon angles.

The uncertainty in the description of the non resonant part, which is quite complicated in the isobar model, has motivated us to fit the data with the RPR model as well, since in this model the background is given in a much simpler form. In the best fit found, which we refer to simply as RPR fit, the resonant content overlaps only partly with that in either BS models or in the RPR-2011A [8] model (only 6 out of 10 nucleon resonances are the same in both cases), the multidipole shape of the form factor is employed and the $\chi^{2} /$ n.d.f. acquires a value of 2.32. Besides other resonances, we have included also a $D_{13}(2120)$ resonance, since its presence leads to a moderate decrease of the $\chi^{2}$ value.

As shown in the subsequent figures, we have achieved a reasonable description of data with the isobar model as well as with the RPR model. Since predictions of both BS models are nearly identical (see Figs. in Ref. [14]), we show only results of the BS1 model and compare them with predictions of the RPR fit, the RPR-2011A [8] model and the well-known isobar KM [11] and SL [7] models.
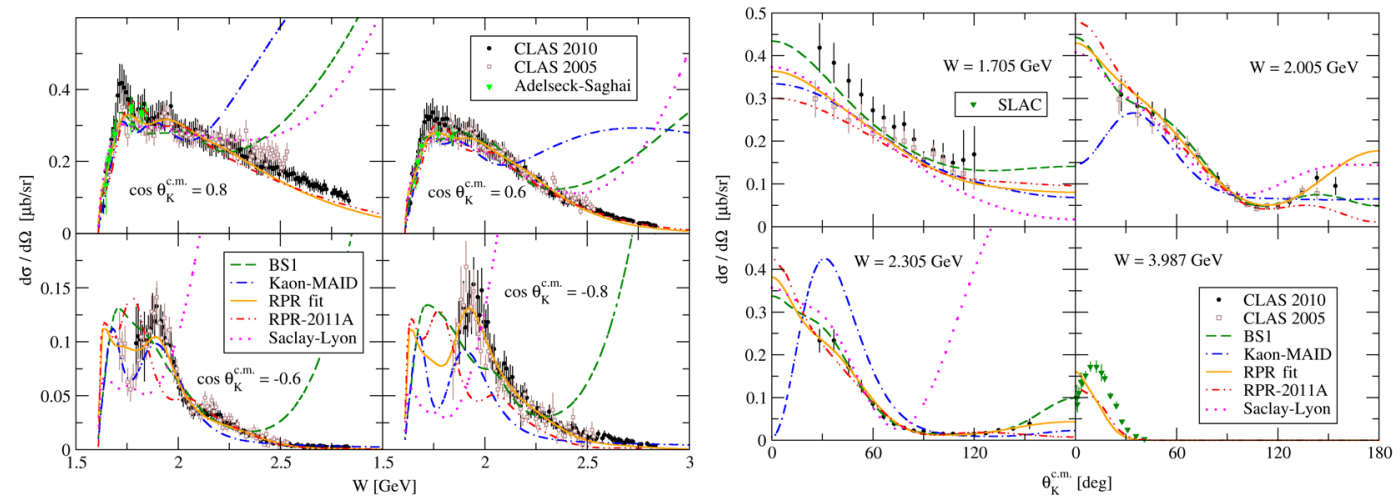

Figure 1. Differential cross section in dependence on the center-of-mass energy $W$ (left) and on the kaon angle $\theta_{K}^{c . m .}$ (right) is shown as predicted by isobar and RPR models. The data stem from CLAS 2005 [4], CLAS 2010 [12], and SLAC [3] collaborations and from the publication of Adelseck and Saghai [1]. Note that the SLAC data may be a subject of reanalysis in the future.

In Fig. 1, we show predictions of differential cross section in dependence either on the energy $W$ or on the kaon angle $\theta_{K}^{c . m}$. computed by several models. There is an ambiguity in the resonance pattern around $1.7 \mathrm{GeV}$ at forward angles (see the left part of Fig. 1): CLAS 2010 data set gives a sharper peak than the CLAS 2005 data, which none of the models displayed captures. The RPR fit is apparently the only model capable of describing the peak around $1.9 \mathrm{GeV}$ at forward angles while both BS1 and BS2 models predict the peak around $1.9 \mathrm{GeV}$ only in the central-angle region. In the forward-angle region, some strength is also apparent around $2.1 \mathrm{GeV}$, which is modelled by 
the high-mass resonances $D_{13}(1875), P_{13}(1900)$, and $F_{15}(2000)$. The RPR fit agrees well with the cross-section data from the threshold up to energies of several $\mathrm{GeV}$ where contributions of nucleon resonances vanish and the contribution of kaon trajectories prevails while the isobar models describe data well only up to $2.4 \mathrm{GeV}$. In the region of backward angles, where the data are not plentiful, there are two broad peaks apparent in the cross section; the one around $2 \mathrm{GeV}$ is quite well captured by the RPR fit. We reckon that this behaviour is achieved by the inclusion of a number of $N^{*}$ 's and we regard the $P_{11}(1880)$ and $D_{13}(1875)$ resonances as the most important ones in this region.

The RPR fit and the BS models give very similar predictions of the angular dependence of the cross section, which differ mainly in the forward- and backward-angle regions (see the right part of Fig. 1). For $\theta_{K}^{c . m .}<40^{\circ}$, the new models predict decreasing angular dependence like the SL and RPR-2011A models, in contrast to the $\mathrm{KM}$ which predicts a very suppressed cross section for $W \geq 2 \mathrm{GeV}$. Note that the consistency of the cross sections in the region of very small kaon angles with the results of the SL model and the fact that these cross sections are dominated by the spin-flip part of the amplitude could predetermine the new models for successful predictions of the cross sections in the production of hypernuclei, similarly to the SL model.

\section{Summary}

The isobar models BS1 and BS2 were constructed using a consistent description for spin-3/2 and spin$5 / 2$ resonances. Spin-3/2 hyperon resonances, whose presence is a novel feature of the isobar model, were found to play an important role in depiction of the background part of the amplitude. The set of nucleon resonances selected in our analysis agrees well with the one selected in the analysis with the RPR model done by the Ghent group. A fit with the RPR model, exploiting the consistent highspin formalism as well, provides a reliable description of data in the resonant and high-spin region. However, the predictions of various models for the cross section at small kaon angles differ and we, therefore, conclude that the data still cannot fix the models fully.

This work was supported by the Grant Agency of the Czech Republic, Grant No. P203/15/04301, and the Grant Agency of the Czech Technical University in Prague, Grant No. SGS16/243/OHK4/3T/14.

\section{References}

[1] R. A. Adelseck, B. Saghai, Phys. Rev. C 42, 108 (1990)

[2] M. Benmerrouche, R. M. Davidson, and N. C. Mukhopadhyay, Phys. Rev. C 39, 2339 (1989)

[3] A. Boyarski et al. (SLAC collaboration), Phys. Rev. Lett 22, 11311133 (1969)

[4] R. Bradford et al. (CLAS collaboration), Phys. Rev. C 73, 035202 (2006)

[5] S. Capstick and W. Roberts, Prog. Part. Nucl. Phys. 45, S241 (2000)

[6] T. Corthals, J. Ryckebusch, and T. Van Cauteren, Phys. Rev. C 73, 045207 (2006)

[7] J. C. David, C. Fayard, G.-H. Lamot, and B. Saghai, Phys. Rev. C 53, 2613 (1996)

[8] L. De Cruz, T. Vrancx, P. Vancraeyveld, and J. Ryckebusch, Phys. Rev. Lett. 108, 182002 (2012)

[9] M. Guidal, J.-M. Laget, and M. Vanderhaeghen, Nucl. Phys. A 627, 645 (1997)

[10] K. A. Olive et al. (Particle Data Group), Chin. Phys. C 38, 090001 (2014)

[11] T. Mart and C. Bennhold, Phys. Rev. C 61, 012201(R) (1999); T. Mart, ibid. 62, 038201 (2000)

[12] M. E. McCracken et al. (CLAS collaboration), Phys. Rev. C 81, 025201 (2010)

[13] V. Pascalutsa, Phys. Rev. D 58, 096002 (1998)

[14] D. Skoupil, P. Bydžovský, Phys. Rev. C 93, 025204 (2016)

[15] T. Vrancx, L. De Cruz, J. Ryckebusch, and P. Vancraeyveld, Phys. Rev. C 84, 045201 (2011) 